Société d'histoire de la révolution de 1848 et des

révolutions du XIXe siècle

Savoirs occultés : du magnétisme à l'hypnose

\title{
Nathalie BRÉMAND, Les socialismes et l'enfance. Expérimentation et utopie (1830-1870)
}

Collection Histoire, Rennes, Presses universitaire de Rennes, 2008, 365

p. ISBN : 978-2-7535-0643-5. 20 euros

\section{Pascale Quincy-Lefebvre}

\section{(2) OpenEdition}

Édition électronique

URL : http://journals.openedition.org/rh19/3885

DOI : $10.4000 /$ rh 19.3885

ISSN : $1777-5329$

Éditeur

La Société de 1848

Édition imprimée

Date de publication : 1 juillet 2009

Pagination : 144-146

ISSN : 1265-1354

Référence électronique

Pascale Quincy-Lefebvre, « Nathalie BRÉMAND, Les socialismes et l'enfance. Expérimentation et utopie (1830-1870) », Revue d'histoire du XIXe siècle [En ligne], 38 | 2009, mis en ligne le 04 septembre 2009, consulté le 22 septembre 2020. URL : http://journals.openedition.org/rh19/3885 ; DOI : https:// doi.org/10.4000/rh19.3885

Ce document a été généré automatiquement le 22 septembre 2020

Tous droits réservés 


\section{Nathalie BRÉMAND, Les socialismes et l'enfance. Expérimentation et utopie (1830-1870)}

Collection Histoire, Rennes, Presses universitaire de Rennes, 2008, 365

p. ISBN : 978-2-7535-0643-5. 20 euros

Pascale Quincy-Lefebvre

1 Cet ouvrage est la version remaniée d'une thèse dirigée par Jean-Noël Luc sur un sujet ayant fait l'objet de publications éparses ou anciennes, d'ailleurs non négligées par l'auteure. L'étude de Nathalie Brémand les dépasse pourtant, autant par son érudition que par la relecture des archives qu'elle propose, au prisme du renouvellement de l'historiographie sur l'enfance. Le livre est alors une mine d'informations mettant parfaitement en lumière le rôle pionnier des socialistes du premier XIX ${ }^{e}$ siècle, à la fois dans l'éclosion d'une nouvelle sensibilité à l'enfance, dans la réflexion sur le statut de l'enfant et dans l'innovation pédagogique. Le mérite du livre est de couvrir un large spectre d'auteurs, de poser la question de l'unité de l'école et du rôle de celle-ci dans l'éclosion d'une nouvelle culture de l'enfance. La facture du livre est classique mais efficace.

Une première partie s'intéresse à la place de l'enfant dans les projets de société développés par des figures bien connues ou moins connues du socialisme dit utopique, comme Étienne Cabet, Charles Fourier, Victor Considerant mais également Albert Laponneray, un "communiste», ou Jules Gay, un "oweniste». L'association est la grande idée à partir de laquelle changer l'ordre social libéral. La révolution doit naître de la réussite et de la dissémination des expériences communautaires. La réorganisation des liens sociaux suppose une place nouvelle faite à l'enfant. Le projet associatif débouche sur une réflexion pédagogique, un creuset d'idées pour une éducation nouvelle de l'enfance régulière ou déviante aux $\mathrm{XIX}^{\mathrm{e}}$ et $\mathrm{XX}^{\mathrm{e}}$ siècles. La première partie de l'ouvrage aborde la question de l'enfant dans le groupe familial ou dans les structures collectives, revient sur le sujet plus connu de son instruction et des sanctions infligées. Plus largement, Nathalie Brémand réexamine la façon de dire, de 
penser le statut de l'enfant. Et l'auteure de rappeler que ces socialistes, tout en évoquant "la dette représentée par l'éducation», ont, avec la théorie de "l'enfant producteur » et, plus encore, avec la représentation de l'enfant comme "personne sociale indépendante » été des pionniers d'une réflexion que l'on pouvait penser plus récente : celle des droits de l'enfant.

Le livre a une autre ambition: confronter théories et réalisations. La seconde partie porte sur l'enfant dans les expérimentations sociales. Elle se subdivise en quatre chapitres. L'auteure distingue les réalisations pour l'enfance, les projets de communautés familiales, puis présente longuement des expériences mieux connues mais qui n'avaient pas été forcément étudiées sous cet angle: l'histoire des communautés icariennes aux États-Unis (1848-1895) et l'histoire fameuse du familistère de Guise (de 1859 à 1880). On peut regretter un déséquilibre dans le traitement au détriment du premier chapitre. Des institutions comme la maison de santé et de sevrage de Beauregard ou bien la maison rurale d'enfance pour l'expérimentation sociétaire de Ry ne font l'objet que de courts développements. La brièveté tient sans doute à la faiblesse des sources, au peu de traces laissées par ces expériences. La connexion est faite avec des travaux sur le mouvement des colonies agricoles ou plus largement le mouvement philanthropique du premier XIX siècle, mais incidemment. Les recherches de Christian Carlier sur cette même période ne sont d'ailleurs pas citées alors que des inspirations sont communes et que des transferts sont réels.

Ce sont les expériences les plus mythiques qui ont le plus retenu l'attention de l'auteure. L'écriture est parfaitement maîtrisée. La lecture est plaisante. Un important travail sur les sources est réalisé. Des pièces intéressantes sont mises au jour, comme par exemple les échanges houleux entre Godin et les représentants de l'Instruction publique, qui sont à verser au dossier d'une histoire du rapport de la République à l'innovation. Les lettres dévoilent toute la complexité des relations entre les tenants de la révolution par le bas et les idéologues ou les administrateurs de l'école de la République. Si le croisement des écrits théoriques avec les sources sur les expérimentations avait été fait par d'autres, il est ici réalisé avec finesse et donne des résultats. L'étude aboutit à relativiser la place de l'enfant dans les expérimentations sociales. Il faut dire que beaucoup ont avorté ou ont eu une vie bien courte, ne permettant pas de réaliser le programme initialement prévu. Les enfants sont bien présents mais ils n'ont guère bénéficié des institutions qui devaient leur être spécifiquement dévolues. Celles-ci ont souvent tardé à se concrétiser et bien souvent elles ont été totalement négligées devant l'urgence de la survie de ces communautés.

Que sont devenus ces enfants? La question est posée mais peu de trajectoires ont pu être reconstituées et on peut se demander si des sources existent pour poursuivre l'enquête. Au final, l'étude révèle toute l'actualité d'un questionnement sur « enfance et changement social " lorsqu'il faut penser la formation de l'individu et l'intérêt du groupe. Sur un mode spécifique chez ces socialistes, mais non sans lien avec d'autres réalisations, la confrontation entre théories et pratiques révèle les tensions associées $\mathrm{au}$ « comment grandir » dans une société moderne. 